\title{
Wild Flowers of Ghyachok VDC, Gorkha District, Nepal
}

\author{
Neera Joshi Pradhan ${ }^{1}$ and Nirmala Joshi \\ ${ }^{1}$ Studio Petals, Khumaltar, Lalitpur, Nepal.E-mail: neera.joshi.pradhan@gmail.com \\ ${ }^{2}$ Communicating author: Department of Plant Resources, Thapathali, \& Society of Community \\ Development Professionals, Kathmandu, Nepal.E-mail:nirmalaktm@gmail.com
}

[Received 28.05.2019; Revised 10.06.2019; Accepted 14.06.2019; Published 30.06.2019]

\begin{abstract}
The present paper describes the wild flowers of Ghyachok in Gorkha District of Nepal. This study area occupies the tropical, subtropical, temperate and sub-alpine vegetation type. The study was conducted during May and early September, 2017. A list of 119 species (dicotyledons 98 , monocotyledons 20 and one gymonsperm) belonging to 54 families and 100 genera were recorded of which 68 species herbs, 22 shrubs, 8 trees, 14 climbers, vine 5 and creeping 2 has been presented. Among 119 wild-flower species, 4 species [Taxus wallichiana, Allium wallichii, Swertia angustifolia, and Arisaema costatum] are endangered and 12 species were reported as rare and protected. This study will be useful to manage the diversity of potential ornamental species and to conserve rare, threatened, endemic and CITES listed plants species of Nepal.
\end{abstract}

Key words: Conservation, Ornamental, Plant diversity, Protected plants

\section{INTRODUCTION}

Nothing can excel the beauty of flowers and the nature. These are the main plant resources of recreational and aesthetic value for human beings. Nepal is rich in its culture and natural resources. There are numerous wild flowering plants in natural habitat, the attractive flowers, fruits, foliage which can be specially used as ornamental plants for the purpose of beauty and pleasant aroma (Joshi 2011). The Himalayan country Nepal is considered as rich in biodiversity due to its own unique climate and varied vegetation from tropical region to temperate and alpine region (NBS 2002).In the past, Hara et al. $(1978,1982)$, Hara and Williams (1979) enumerated 5067 species of flowering plants of Nepal. Well over 6000 species of flowering plants has so far been recorded from Nepal (Press et al. 2002). The floral diversity of Nepal includes 6973 species of Angiosperms (UNEP-WCMC 2004). However, according to latest publication, there are 5500 species of flowering plants (Rajbhandari 2015). Among the 5500 species of flowering plants, 324 species are endemic to Nepal (Rajbhandari et al. 2016). Most of the beautiful ornamental flowers have come from the wild which exist in natural habitat. But the population structure of most of them are decreasing in natural habitat due to different types of calamities such as forest fire, flood, landslides, erosion, infrastructure development, introducing of invasive species, lack of conservation awareness knowledge and exploitation etc. Therefore several such important plants have become endangered and finally led to extinction (Shrestha \& Joshi 1996).

In this context, however such plants have not been fully described in illustrative form in modern botany. Today the role of education of botanical art and illustration is one of the powerful creative tool as visual communication to bring the awareness to explore, research and conserve such rich biodiversity of the natural heritage. The objective of the present study is to explore and describe scientifically the beautiful native, important wild flowers 
distributed wildly within the area and document them both in possible illustrative form and prepare checklist.

\section{Study area}

Nepal is located in the central Himalayas and occupies $147,181 \mathrm{~km}^{2}$ area The country is situated between latitudes $26^{\circ} 22^{\prime}$ and $30^{\circ} 27^{\prime} \mathrm{N}$ and longitudes $80^{\circ} 40^{\prime}$ and $88^{\circ} 12^{\prime} \mathrm{E}$. The country has 14 zones and 77 districts comprised within 7 provinces. Among them, Gorkha district lies in Gandaki zone of Gandaki Pradesh of Province No.4. Geographically the Gorkha district coordinates $28^{\circ} .20^{\prime}$ latitude and $84^{\circ} .71^{\prime}$ longitude. The district covers an area $3616 \mathrm{~km}^{2}$. The elevation of the district ranges from $228 \mathrm{~m}$ to $8163 \mathrm{~m}$ above mean sea level. Its boundaries are surrounded by Dhading in the east, Tanhu, Lamjung and Manang in the west, Manang north then Chitwan and Tanahu in the south Gorkha district comprises nine rural and two metropolitan municipalities. The district consists of 94 Wards (DPG 2017).

The present study was carried out in Ghyachok VDC (Village Development Committee) that is placed under Ajirkot rural municipality, Wards 1-2 (Figure 1). Ghyachok is the nearest village from Barpak, was an epicenter hit by devastating earthquake April 2015, which caused massive damages to its people and places. Ghyachok is located in between latitude $28^{\circ} 40^{\prime} 10^{\prime \prime}$ and $84^{\circ} 41^{\prime} 58^{\prime \prime}$ E longitude. The altitude of this VDC ranges from $1200 \mathrm{~m}$ to $3381 \mathrm{~m}$. It is rich in its cultural diversity with various ethnic groups, $34.57 \%$ Gurungs, Brahmins 21.08\% and Tamangs $12.92 \%$ (DPG 2017). The average rainfall of this area is reported as $223.49 \mathrm{~mm}$ in June- September , 2017. Minimum Temperature in Summer is maximum $32.7^{\circ} \mathrm{C}$ while in winter is minimum $8.1^{\circ} \mathrm{C}, 2017$ in Gorkha Municipality and snowy most of the time above $1750 \mathrm{~m}$ Ajirkot Rural municipality in winter season (DHM 2017).

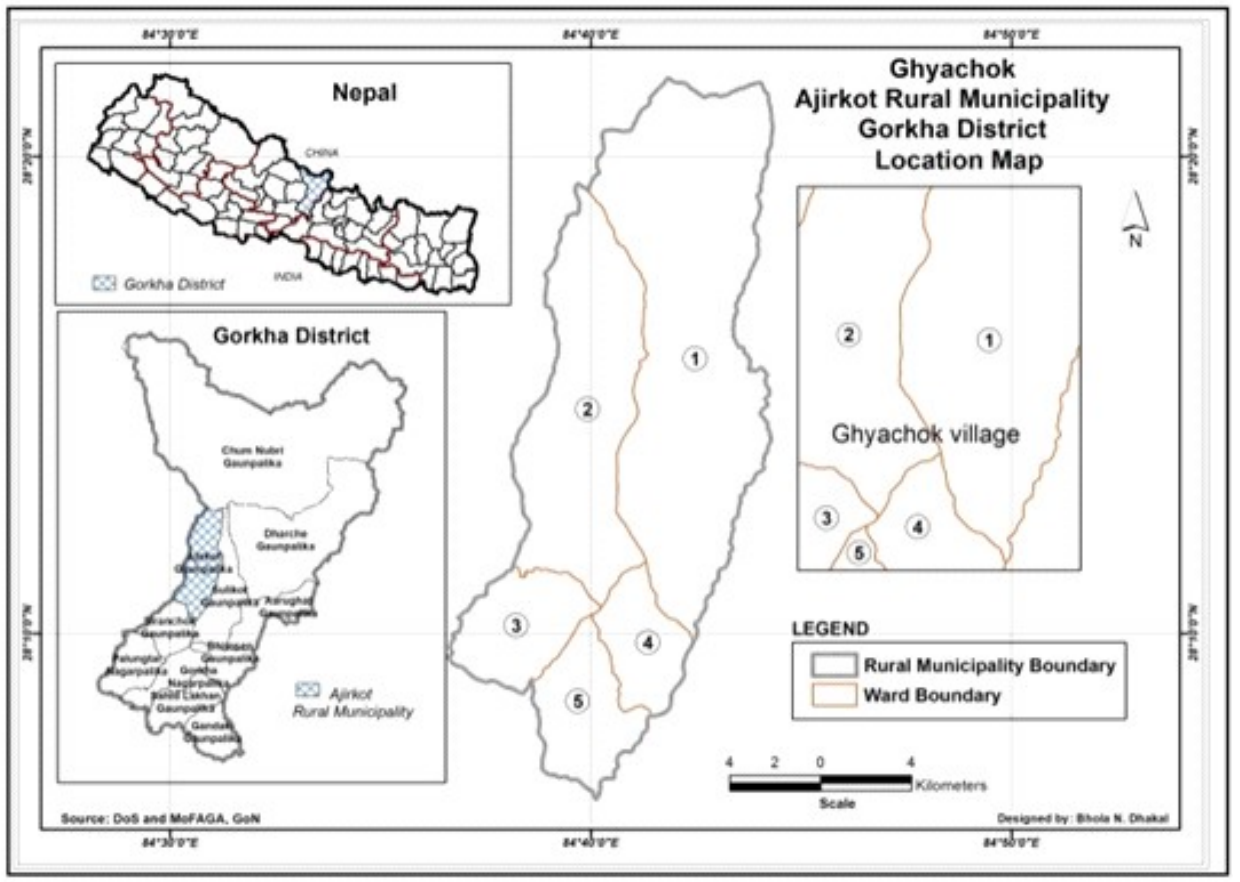

Figure 1. Location map of study sites of Ghyachok VDC 
It covers subtropical and temperate to subalpine zone from $1200 \mathrm{~m}$ to above 3000 min terms of vegetation. The vegetation types of Ghyachok Village are (i) Schima wallichiiAlnus nepalensis forest (between 1200-1700m); (ii) Daphniphyllum - Myrsine mixed forest is in between $1700-2100 \mathrm{~m}$, is extensively widespread; (iii) Daphniphyllum Rhododendron forest in between $2100-2300 \mathrm{~m}$ range is associated with many orchids including Dendrobium eriflorum, Coelogyne nitida etc.; (iv) Rhododendron - Laurel forest between 2300 - 2600 m; Rhododendron arboreum, Alnus nepalensis, Berberis, Viburnum erubescens, Marsdenia sp. etc. are the associated species where as among orchids, Pleione hookeriana, Coelogyne sp. Dendrobium species are found; (v) Rhododendron arboreum forest between $2600-3100 \mathrm{~m}$ only Rhododendron arboreum is dominant. Among Taxus wallichiana, Lyonia ovalifolia, and Berberis species. Rhododendron arboreum are found dominant upto $3100 \mathrm{~m}$. Along with Rhododendron arboreum, Taxus wallichiana, Berberis spp. are also found associated; (vi) Above 3100 $\mathrm{m}$ the tree-line is occupied by shrubs of Berberis spp., small trees of Rhododendron arboretum, Abies sp., Prunus sp, Anemone obtisiloba, Fragaria nubicula, Potentilla sp. and Bistorta sp.

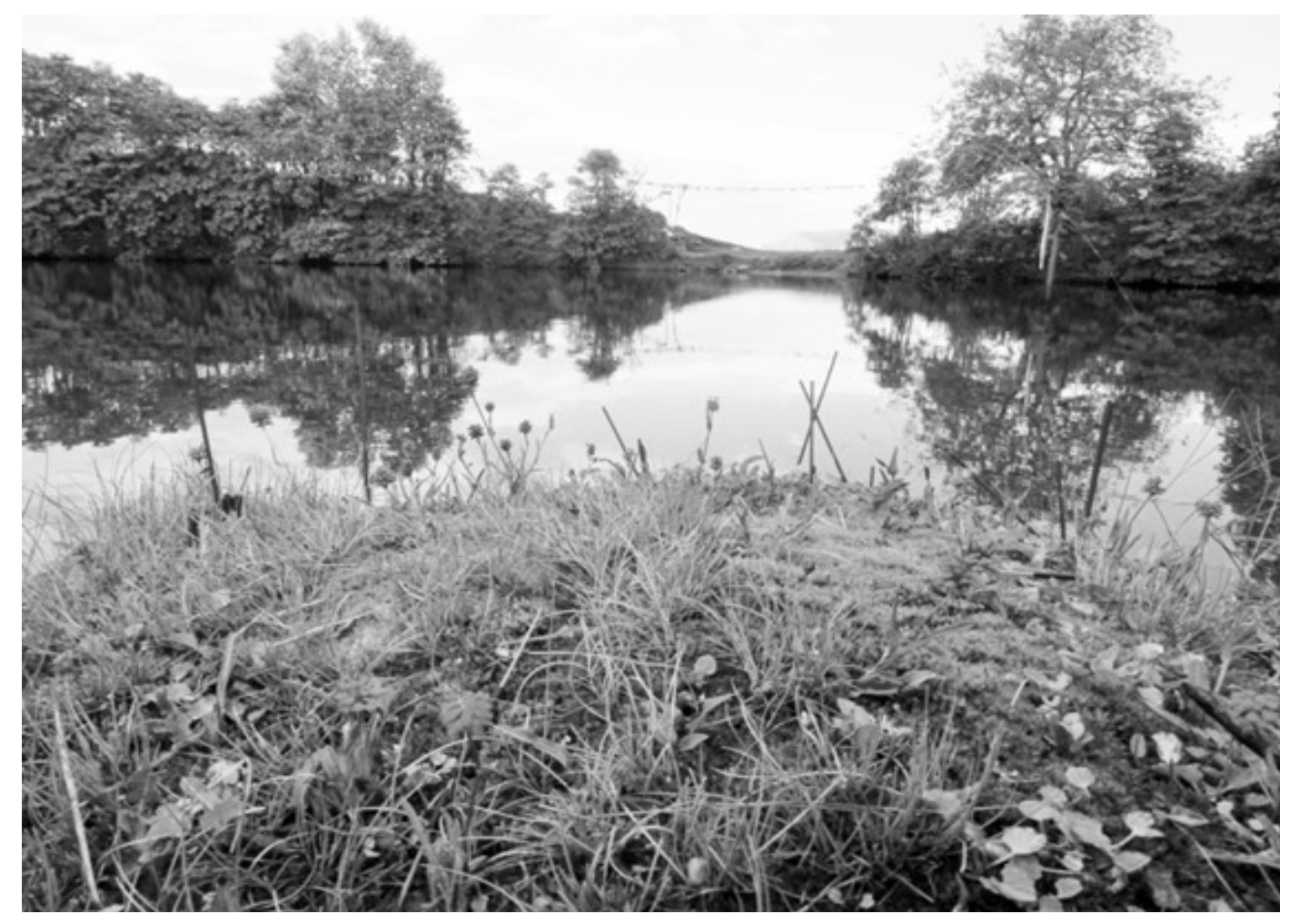

Figure 2. Vegetation of Nagay Ra Pond area

At $3250 \mathrm{~m}$, the Nagay Ra Pond (Ra means pond in Gurung language) is located and is a Hindu pilgrimage and is worshipped on every full-moon of August. The Pond is the source of drinking water for the shepherds. The range of Himalayas Manaslu (known as Himalchuli), Lamjung Himal, Boudha Himal, Rubinala pass, Ganesh Himal and Langtang are visible from the NagayRa. After Nagay Pokhari there are few small ponds on the way to Ram Bhanjyang $3260 \mathrm{~m}$ and Deurali $3360 \mathrm{~m}$ (Figure 2). 


\section{MATERIALS AND METHODS}

The study area, Ghyachok VDC was selected firstly due to its virgin vegetation pending any scientific study on its rich biodiversity, historical importance, cultural richness and altitudinal variation. The objective of study and research were to prepare a checklist of the wild flora of Ghyachok VDC, Gorkha district in Nepal. Based on the altitudinal variations, plants were collected from lowland, midland and highland for understanding the phenology changes. The lowland (tropical) sites were selected near Baluwa Bazar $921 \mathrm{~m}$, midland (subtropical sites) were Sallaghari 1050m, Milinge 1106m, Kaldobesi 1267m Dhrapani, Ghyachok 1700, Dhodke Pokhari 1800m to Chihan Danda; temperate sites were Dhaulo Danda 2067m, Dobato Kharka 2278m, Bag Khor 2475m, Podu Chuatara 2537, Najyu Kharka 2910m, and Bisauni $3092 \mathrm{~m}$. The high land or subalpine sites includes way to Nagay Pokhari 3250m, Ram Bhanjyang $3264 \mathrm{~m}$ to Deurali 3360m. Location Map of study sites Ghyachok village is shown in Figure 1.

Collection of specimens were carried out in two different seasons of blooming period of the flowering plants in May and early September, 2017. During the two field visits, the plants were collected from different habitats such as forest, roadsides and fallow land. Habit, habitat, locality, altitude, flower colour and flowering time were recorded in Field Note Book. The epiphytes-are rare in the study sites, however, were also noted in Field Note Book and were photographed. At the same time, field drawings and sketching of habit, habitat and morphological structures of the epiphytes and some attractive plants were also executed in the Field Sketch Book. The plants were collected, tagged and pressed in blotters for drying following Jain and Rao (1977).

The collected specimens were identified in the field with local people's information and by consulting relevant references (Hara \& Williams, 1979; Hara et al. 1978; 1982; Shrestha et al. 2018; Stainton \& Polunin 1984; Stainton 1988; Watson et al. 2011). Pearce \& Cribb (2002), Rajbhandari \& Bhattarai (2001) and Raskoti (2009) were used as references for the identification of Orchids. The collected specimens were also cross-checked with authentic voucher specimens housed in the National Herbarium and Plant Laboratories (KATH) for confirmation. Nomenclature of the collected plant species and the six families such as Compositae (Asteraceae), Cruciferae (Brassicaceae), Labiatae (Lamiaceae), Leguminosae (Fabaceae) and Gramineae (Poaceae) are followed according to www.theplantlist.org and Rajbhandari et al. (2011, 2012).

The genera and species are enumerated alphabetically within the family (Table 1). The habit, locality, altitude, habitat, flower colour and flowering time were also incorporated. The collected herbarium specimens are deposited at KATH Herbarium.

Finally the completed morphological structures in pen and ink botanical illustrations (scientific line drawings) of 2 specimens of epiphytes i.e. Pleione hookeriana (Orchidaceae) and Rhododendron dalhousiae (Ericaceae) are presented in Figures $5 \& 6$.

The status of collected plants such as endemic, threatened, endanger, CITES Appendix are recorded from published references (CAMP 2001; Ghimire et al. 2008, Joshi et al. 2017). Abundance and/or rarity of a plants species are deremined from the visual field observation (Table 1).

\section{RESULTS AND DISCUSSION}

The documentation of wild flowers in Ghyachok VDC (Village Development Committee) of the Gorkha district of Nepal showed the attractive rich floral diversity. The wild flowers are mainly based on their habit, habitat, morphological structure, attractive flower colour, fruits, foliage and plants with their aroma (Joshi 2011). 
A total of 119 species of flowering plants, belonging to 54 families and 100 genera were collected, of which 118 species Angiosperms and one species Gymnosperm has been presented.

Among the studied species, dicotyledons were the most common groups distributed with 45 families consist of 98 species, monocotyledons in 8 families with 20 species and gymnosperm just one species. This study indicated that the maximum wild flowers are with the dicotyledons in comparison to monocots and gymnosperm.

The dominant families in the present study are Orchidaceae with 11 species, followed by Ranunculaceae and Rosaceae 9 species each, Compositae 8 species, Leguminosae with 7 species, Ericaceae and Polygonaceae 4 species each, Apocynaceae, Begoniaceae, Berberidaceae, Gentianaceae, Melastomataceae 3 species each and rest of families consist of 2 or 1 species (Figure 3; Table 1).

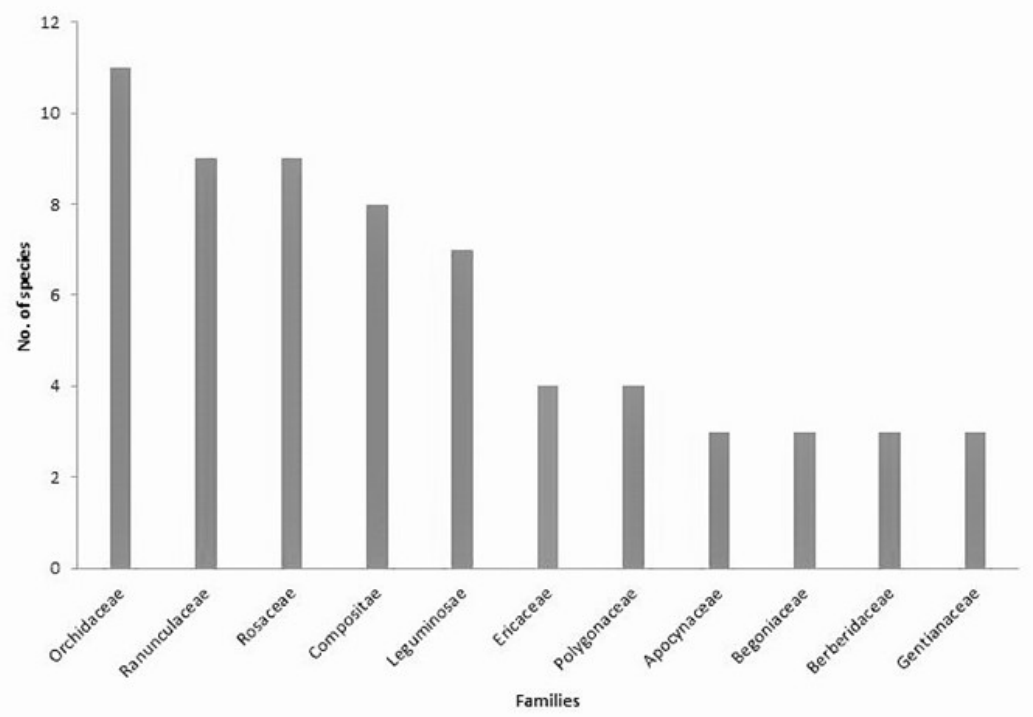

Figure 3. Dominant families of the study area

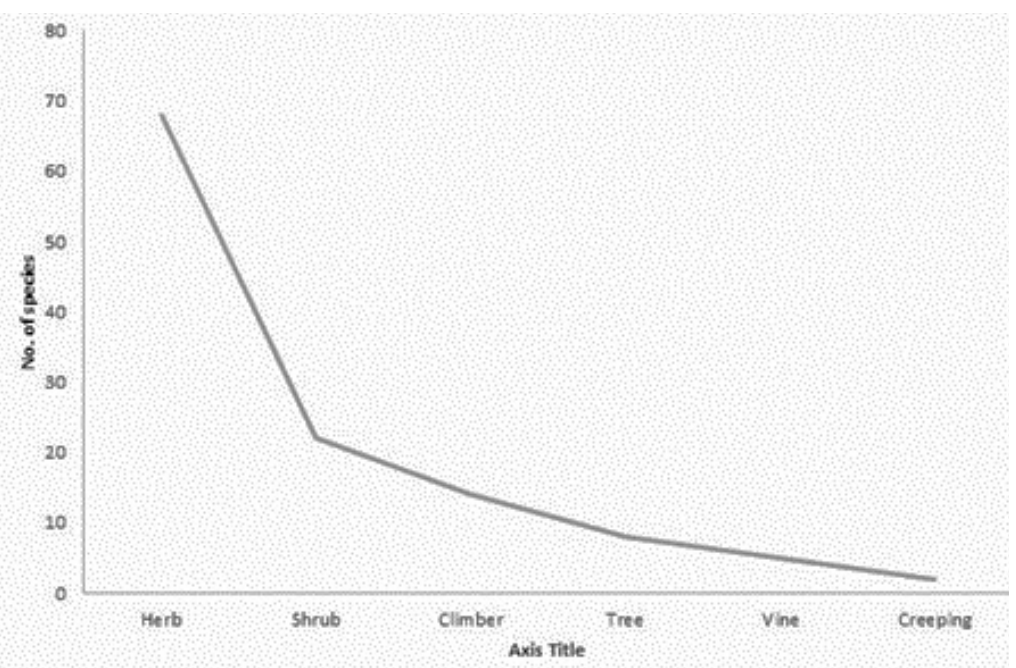

Figure 4. Habit-group representation of the collected wild flowers 
The dominant genera in the recorded flora are Anaphalis, Begonia, Desmodium and Rubus with 3 species each followed by the second large genera with 2 species are Aster, Berberis, Clematis, Codonopsis, Dendrobium, Gaultheria, Hypericum, Impatiens, Rhododendron, Saxifraga and Thalictrum (Table 1).

Based on habit the enumerated wild flowers, maximum number of species were herbs 68 species, followed by 22 species shrubs, 14 species climbers, 8 species trees, creeping and vines 2 species each (Figure 4).

The present study recorded one endemic species to Nepal, Delphinium himalayae from the way to Nagpokhari at 3189 m altitude (Shrestha \& Joshi 1996; Ghimire et al. 2008; Rajbhandari \& Adhikari 2009).

Table 1. Checklist of wild flowers of Ghychok VDC, Gorkha district of Nepal including scientific name, family, locality, altitude (m), habit, flower colour, flowering time, collector no. status [Abbreviations used: $\mathrm{A}=$ Abundant; $\mathrm{CE}=$ Critically Endangered; $\mathrm{T}=$ Threatened; $\mathrm{R}$ $=$ Rare $; \mathrm{E}=$ Endemic $; \mathrm{HE}=$ Himalaya Endemic; App.II = CITES Appendix II $]$

\begin{tabular}{|c|c|c|c|c|c|c|c|}
\hline Scientific name & Locality & $\begin{array}{l}\text { Altitud } \\
\text { e (m) }\end{array}$ & Habit & $\begin{array}{l}\text { Flower } \\
\text { colour }\end{array}$ & Flowering time & $\begin{array}{l}\text { Collec- } \\
\text { tion } \\
\text { no. }\end{array}$ & Status \\
\hline $\begin{array}{l}\text { Aeschynanthus parviflorus (D.Don) } \\
\text { Spreng. [Gesneriaceae] }\end{array}$ & Dharapani & 1280 & Herb & Orange & July-September & GG135 & A \\
\hline $\begin{array}{l}\text { Allium wallichii Kunth } \\
\text { [Amaryllidaceae] }\end{array}$ & Deurali & 3278 & Herb & Purple & July- October & GG122 & $\mathrm{T}$ \\
\hline Alnus nepalensis D.Don [Betulaceae] & Ghyachchok & 1686 & Tree & Brown & $\begin{array}{l}\text { September- } \\
\text { October }\end{array}$ & GG80 & A \\
\hline $\begin{array}{l}\text { Anaphalis busua (Buch.-Ham.ex D. } \\
\text { Don) DC. [Compositae] }\end{array}$ & $\begin{array}{l}\text { Dhaunlo- } \\
\text { danda }\end{array}$ & 2067 & Herb & White & $\begin{array}{l}\text { May - } \\
\text { September }\end{array}$ & GG84 & $\bar{A}$ \\
\hline $\begin{array}{l}\text { Anaphalis contorta (D.Don) Hook.f. } \\
\text { [Compositae] }\end{array}$ & $\begin{array}{l}\text { Milinge } \\
\text { village }\end{array}$ & 1635 & Herb & White & $\begin{array}{l}\text { May - } \\
\text { September }\end{array}$ & GG70 & A \\
\hline $\begin{array}{l}\text { Anaphalis triplinervis (Sims) C.B. } \\
\text { Clarke [Compositae] }\end{array}$ & $\begin{array}{l}\text { Dobato } \\
\text { Kharka to } \\
\text { Bagkhor }\end{array}$ & 2358 & Herb & White & $\begin{array}{l}\text { May - } \\
\text { September }\end{array}$ & GG95 & $\mathrm{A}$ \\
\hline $\begin{array}{l}\text { Anemone obtusiloba } \text { D. Don } \\
\text { [Ranunculaceae] }\end{array}$ & $\begin{array}{l}\text { Damara, } \\
\text { Ghyachok }\end{array}$ & 2443 & Herb & White & $\begin{array}{l}\text { May - } \\
\text { September }\end{array}$ & GG29 & $\mathrm{A} / \mathrm{HE}$ \\
\hline $\begin{array}{l}\text { Aralia leschenaultii (DC.) J.Wen } \\
\text { [Araliaceae] }\end{array}$ & $\begin{array}{l}\text { Belduchheldu } \\
\text {, Ghyachowk }\end{array}$ & 2011 & Vine & Green & April -My & GG17 & A \\
\hline $\begin{array}{l}\text { Argyreia hookeri C.B.Clarke } \\
\text { [Convolvulaceae] }\end{array}$ & Saalghari & 1037 & Climber & Purple & $\begin{array}{l}\text { September- } \\
\text { October }\end{array}$ & GG67 & $\bar{A}$ \\
\hline $\begin{array}{l}\text { Arisaema costatum (Wall.) Mart. ex } \\
\text { Schott [Araceae] }\end{array}$ & $\begin{array}{l}\text { Chiyandanda, } \\
\text { Ghyachowk } \\
\text { village }\end{array}$ & 1910 & Herb & $\begin{array}{l}\text { Dark } \\
\text { purple }\end{array}$ & May -June & GG14 & $\mathrm{R} / \mathrm{H} \mathrm{E}$ \\
\hline $\begin{array}{l}\text { Aster albescens (DC.) Koehne } \\
\text { [Compositae] }\end{array}$ & $\begin{array}{l}\text { Way to } \\
\text { Nagepokhri }\end{array}$ & 3037 & Shrub & Purple & $\begin{array}{l}\text { September- } \\
\text { October }\end{array}$ & GG110 & $\mathrm{A}$ \\
\hline $\begin{array}{l}\text { Aster himalaicus C.B.Clarke } \\
\text { [Compositae] }\end{array}$ & $\begin{array}{l}\text { Way to } \\
\text { Nagepokhri }\end{array}$ & 3189 & Herb & Blue & $\begin{array}{l}\text { September- } \\
\text { October }\end{array}$ & GG111 & $\mathrm{A}$ \\
\hline $\begin{array}{l}\text { Bahunia vahlii Wight \& Arn. } \\
\text { [Leguminosae] }\end{array}$ & $\begin{array}{l}\text { Salghari: } \\
\text { Between } \\
\text { Baluwa and } \\
\text { Kaldobensi }\end{array}$ & 1050 & Vine & White & March-May & GG1 & $\mathrm{A}$ \\
\hline $\begin{array}{l}\text { Begonia josephii A.DC. } \\
\text { [Begoniaceae] }\end{array}$ & $\begin{array}{l}\text { On the way to } \\
\text { Dobato } \\
\text { Kharka }\end{array}$ & 2358 & Herb & White & $\begin{array}{l}\text { September- } \\
\text { October }\end{array}$ & GG105 & $\mathrm{R}$ \\
\hline Begonia picta $\mathrm{Sm}$. [Begoniaceae] & Saalghari & 1066 & Herb & Pink & August-October & GG69 & A \\
\hline $\begin{array}{l}\text { Begonia rubella Buch.-Ham. ex D. } \\
\text { Don [Begoniaceae] }\end{array}$ & $\begin{array}{l}\text { On the way to } \\
\text { Bagkhor }\end{array}$ & 2418 & Herb & White & $\begin{array}{l}\text { September- } \\
\text { October }\end{array}$ & GG102 & $\mathrm{R} / \mathrm{HE}$ \\
\hline $\begin{array}{l}\text { Berberis chitria } \text { Buch.-Ham. ex D. } \\
\text { Don [Berberidaceae] }\end{array}$ & $\begin{array}{l}\text { Between } \\
\text { Dhodke } \\
\text { pokhari and } \\
\text { Ghyachowk }\end{array}$ & 1817 & Shrub & Yellow. & April -June & GG24 & A \\
\hline
\end{tabular}


Neera Joshi Pradhan \& Nirmala Joshi 47

\begin{tabular}{|c|c|c|c|c|c|c|c|}
\hline Scientific name & Locality & $\begin{array}{l}\text { Altitud } \\
\text { e (m) }\end{array}$ & Habit & $\begin{array}{l}\text { Flower } \\
\text { colour }\end{array}$ & Flowering time & $\begin{array}{l}\text { Collec- } \\
\text { tion } \\
\text { no. }\end{array}$ & Status \\
\hline $\begin{array}{l}\text { Berberis wallichiana DC. } \\
\text { [Berberidaceae] }\end{array}$ & $\begin{array}{l}\text { Between naag } \\
\text { Pokhari } \\
\text { Kharka and } \\
\text { Najyu kharka }\end{array}$ & 2910 & Shrub & $\begin{array}{l}\text { Greenis } \\
\mathrm{h} \\
\text { Yellow }\end{array}$ & April -June & GG48 & $\mathrm{A}$ \\
\hline $\begin{array}{l}\text { Bistorta amplexicaulis (D. Don) } \\
\text { Greene [Polygonaceae] }\end{array}$ & $\begin{array}{l}\text { Way to } \\
\text { Nagepokhri }\end{array}$ & 3189 & Herb & $\begin{array}{l}\text { Dark } \\
\text { Pink } \\
\end{array}$ & July -september & GG113 & $\mathrm{A}$ \\
\hline $\begin{array}{l}\text { Boehmeria rugulosa Wedd. } \\
\text { [Urticaceae] }\end{array}$ & $\begin{array}{l}\text { Saalghari to } \\
\text { Milinge }\end{array}$ & 1428 & Tree & $\begin{array}{l}\text { Browni } \\
\text { sh } \\
\text { White }\end{array}$ & $\begin{array}{l}\text { August- } \\
\text { September }\end{array}$ & GG76 & $\mathrm{A}$ \\
\hline $\begin{array}{l}\text { Boenninghausenia albiflora (Hook.) } \\
\text { Rchb. es Meisn. [Rutaceae] }\end{array}$ & Saalghari & 1037 & Herb & White & April-May & GG62 & $\mathrm{A}$ \\
\hline Caltha palustris L. [Ranunculaceae] & $\begin{array}{l}\text { Near Nage } \\
\text { Pokhari }\end{array}$ & 3250 & Herb & Yellow & April-May & GG34 & A \\
\hline $\begin{array}{l}\text { Ceropegia pubescens Wall. } \\
\text { [Apocynaceae] }\end{array}$ & $\begin{array}{l}\text { On the way to } \\
\text { Dobato } \\
\text { Kharka }\end{array}$ & 2278 & Climber & $\begin{array}{l}\text { Greenish } \\
\text { Yellow }\end{array}$ & July-October & GG90 & $\mathrm{R}$ \\
\hline $\begin{array}{l}\text { Chlorophytum nepalense (Lindl.) } \\
\text { Baker [Asparagaceae] }\end{array}$ & $\begin{array}{l}\text { On the way to } \\
\text { Bagkhor }\end{array}$ & 2458 & Herb & White & July-September & GG107 & $\mathrm{R}$ \\
\hline $\begin{array}{l}\text { Clematis acuminata DC. } \\
\text { [Ranunculaceae] }\end{array}$ & $\begin{array}{l}\text { Rambhanjyan } \\
\mathrm{g}\end{array}$ & 3261 & Climber & White & July-October & GG128 & $\mathrm{A}$ \\
\hline $\begin{array}{l}\text { Clematis montana Buch.-Ham.ex DC. } \\
\text { [Ranunculaceae] }\end{array}$ & $\begin{array}{l}\text { Between } \\
\text { Naag Pokhari } \\
\text { Kharka and } \\
\text { Baagkhor }\end{array}$ & 3085 & Climber & $\begin{array}{l}\text { Pinkish } \\
\text { White }\end{array}$ & April -May & GG33 & $\mathrm{A}$ \\
\hline $\begin{array}{l}\text { Codonopsis thalictrifolia Wall. } \\
\text { [Campanulaceae] }\end{array}$ & Deurali & 3318 & Herb & Blue & July- september & GG125 & $\mathrm{A}$ \\
\hline $\begin{array}{l}\text { Codonopsis viridis Wall. } \\
\text { [Campanulaceae] }\end{array}$ & $\begin{array}{l}\text { On the way to } \\
\text { Bagkhor }\end{array}$ & 2418 & Climber & $\begin{array}{l}\text { Purplis } \\
\text { h Green }\end{array}$ & $\begin{array}{l}\text { September- } \\
\text { November }\end{array}$ & GG101 & $\mathrm{R}$ \\
\hline $\begin{array}{l}\text { Coelogyne nitida (Wall. ex D. Don) } \\
\text { Lindl. [Orchidaceae] }\end{array}$ & $\begin{array}{l}\text { Gangrogo } \\
\text { kharka }\end{array}$ & 1963 & Herb & White & April -June & Photo & App.II \\
\hline $\begin{array}{l}\text { Corydalis leptocarpa Hook.f. \& } \\
\text { Thomsom [Papaveraceae] }\end{array}$ & $\begin{array}{l}\text { Belduchheldu } \\
\text {, Ghyachowk }\end{array}$ & 2055 & Herb & Purple, & May -August & GG18 & $\mathrm{A}$ \\
\hline $\begin{array}{l}\text { Cotoneaster microphyllus Wall. ex } \\
\text { Lindl. [Rosaceae] }\end{array}$ & $\begin{array}{l}\text { Near } \\
\text { Poduchautara }\end{array}$ & 2688 & Shrub & White & April- May & GG52 & $\mathrm{A}$ \\
\hline $\begin{array}{l}\text { Crotalaria prostata } \text { Rottl. ex Willd. } \\
\text { [Leguminosae] }\end{array}$ & $\begin{array}{l}\text { Saalghari to } \\
\text { Milinge }\end{array}$ & 1428 & Herb & Yellow & $\begin{array}{l}\text { September- } \\
\text { October }\end{array}$ & GG74 & $\mathrm{A}$ \\
\hline $\begin{array}{l}\text { Dactylicapnos scandens (D.Don) } \\
\text { Hutch. [Papaveraceae] }\end{array}$ & $\begin{array}{l}\text { Near Milinge } \\
\text { village, } \\
\text { Ghyachowk }\end{array}$ & 1491 & Climber & Yellow. & April -June & GG57 & $\mathrm{A}$ \\
\hline $\begin{array}{l}\text { Delphinium himalayae Munz } \\
\text { [Ranunculaceae] }\end{array}$ & $\begin{array}{l}\text { Way to } \\
\text { Nagepokhri }\end{array}$ & 3189 & Herb & Blue & $\begin{array}{l}\text { August- } \\
\text { September }\end{array}$ & Photo & $\mathrm{E} /$ \\
\hline $\begin{array}{l}\text { Dendrobium eriiflorum Griff. } \\
\text { [Orchidaceae] }\end{array}$ & $\begin{array}{l}\text { Damara, } \\
\text { Ghyachok }\end{array}$ & 2383 & Herb & $\begin{array}{l}\text { Cream } \\
\text { Yellow }\end{array}$ & April-June & Photo & App.II \\
\hline $\begin{array}{l}\text { Dendrobium moniliforme (L.) } \\
\text { Sw. [Orchidaceae] }\end{array}$ & $\begin{array}{l}\text { Damara, } \\
\text { Ghyachok }\end{array}$ & 2383 & Herb & White & April -June & Photo & App.II \\
\hline $\begin{array}{l}\text { Desmodium confertum DC. } \\
\text { [Leguminosae] }\end{array}$ & $\begin{array}{l}\text { Milinge to } \\
\text { Ghyachchok }\end{array}$ & 1635 & Shrub & Purple & $\begin{array}{l}\text { August- } \\
\text { September }\end{array}$ & GG72 & $\mathrm{A}$ \\
\hline $\begin{array}{l}\text { Desmodium elegans DC. } \\
\text { [Leguminosae] }\end{array}$ & $\begin{array}{l}\text { Milinge to } \\
\text { Ghyachchok }\end{array}$ & 1673 & Shrub & Purple & August-october & GG136 & A \\
\hline $\begin{array}{l}\text { Desmodium heterocarpon (L.) DC. } \\
\text { [Leguminosae] }\end{array}$ & Milinge & 1405 & Herb & Purple & $\begin{array}{l}\text { August- } \\
\text { September }\end{array}$ & GG5 & $\mathrm{A}$ \\
\hline $\begin{array}{l}\text { Dichroa febrifuga Lour. } \\
\text { [Hydrangeaceae] }\end{array}$ & $\begin{array}{l}\text { On the way to } \\
\text { Dobato } \\
\text { Kharka }\end{array}$ & 2278 & Shrub & Blue & August-October & GG88 & $\mathrm{A}$ \\
\hline $\begin{array}{l}\text { Dipsacus inermis Wall. } \\
\text { [Caprifoliaceae] }\end{array}$ & $\begin{array}{l}\text { Way to } \\
\text { Nagepokhri }\end{array}$ & 3189 & Herb & White & $\begin{array}{l}\text { September- } \\
\text { October }\end{array}$ & GG118 & A \\
\hline $\begin{array}{l}\text { Disporum cantoniense (Lour.) Merr. } \\
\text { [Colchicaceae] }\end{array}$ & $\begin{array}{l}\text { Between } \\
\text { Dhodke } \\
\text { pokhari and } \\
\text { Ghyachowk }\end{array}$ & 1817 & Herb & $\begin{array}{l}\text { Light } \\
\text { Green. }\end{array}$ & April -June & GG25 & $\mathrm{R}$ \\
\hline $\begin{array}{l}\text { Dubyaea hispida (D.Don) DC. } \\
\text { [Compositae] }\end{array}$ & Deurali & 3318 & Herb & Yellow & $\begin{array}{l}\text { August- } \\
\text { September }\end{array}$ & GG126 & $\mathrm{A}$ \\
\hline
\end{tabular}




\begin{tabular}{|c|c|c|c|c|c|c|c|}
\hline Scientific name & Locality & $\begin{array}{l}\text { Altitud } \\
\text { e (m) }\end{array}$ & Habit & $\begin{array}{l}\text { Flower } \\
\text { colour }\end{array}$ & Flowering time & $\begin{array}{l}\text { Collec- } \\
\text { tion } \\
\text { no. }\end{array}$ & Status \\
\hline $\begin{array}{l}\text { Erigeron trilobus (Decne.) Boiss. } \\
\text { [Compositae] }\end{array}$ & $\begin{array}{l}\text { Milinge to } \\
\text { Ghyachchok }\end{array}$ & 1635 & Herb & Yellow & $\begin{array}{l}\text { August- } \\
\text { September }\end{array}$ & GG75 & $\mathrm{A}$ \\
\hline $\begin{array}{l}\text { Euonymus echinatus Wall. } \\
\text { [Celastraceae] }\end{array}$ & $\begin{array}{l}\text { Near Najyu } \\
\text { Kharka }\end{array}$ & 2761 & Shrub & $\begin{array}{l}\text { Light } \\
\text { Green }\end{array}$ & May -June & GG49 & $\mathrm{R}$ \\
\hline $\begin{array}{l}\text { Euphorbia wallichii Hook.f. } \\
\text { [Ephorbiaceae] }\end{array}$ & $\begin{array}{l}\text { Between naag } \\
\text { Pokhari } \\
\text { Kharka and } \\
\text { Bisauni }\end{array}$ & 3081 & Herb & Yellow & April-June & GG45 & A \\
\hline $\begin{array}{l}\text { Fagopyrum tataricum (L.) Gaertn. } \\
\text { [Polygonaceae] }\end{array}$ & $\begin{array}{l}\text { Ghyachowk } \\
\text { village }\end{array}$ & 1695 & Herb & White & April-June & GG9 & $\mathrm{A}$ \\
\hline $\begin{array}{l}\text { Fragaria nubicola (Lindl. ex Hook.f.) } \\
\text { Lacaita [Rosaceae] }\end{array}$ & $\begin{array}{l}\text { Between naag } \\
\text { Pokhari } \\
\text { Kharka and } \\
\text { Bisauni }\end{array}$ & 3092 & Herb & White & April-June & GG42 & $\mathrm{A}$ \\
\hline $\begin{array}{l}\text { Gaultheria trichophylla Royle } \\
\text { [Ericaceae] }\end{array}$ & $\begin{array}{l}\text { Near Nage } \\
\text { Pokhari }\end{array}$ & 3250 & Herb & Pink & April-June & GG41 & $\mathrm{A}$ \\
\hline $\begin{array}{l}\text { Gaultheria nummularioides D.Don } \\
\text { [Ericaceae] }\end{array}$ & $\begin{array}{l}\text { Dhaunlo- } \\
\text { danda }\end{array}$ & 2067 & $\begin{array}{l}\text { Creepi } \\
\text { ng on } \\
\text { ground }\end{array}$ & White & $\begin{array}{l}\text { September- } \\
\text { October }\end{array}$ & GG86 & $\mathrm{A}$ \\
\hline $\begin{array}{l}\text { Gentiana ornata (D.Don) Wall. ex } \\
\text { Griseb. [Gentianaceae] }\end{array}$ & Deurali & 3278 & Herb & Purple & $\begin{array}{l}\text { September- } \\
\text { october }\end{array}$ & GG129 & $\mathrm{R}$ \\
\hline $\begin{array}{l}\text { Geranium lambertii Sweet } \\
\text { [Geraniaceae] }\end{array}$ & $\begin{array}{l}\text { On the way to } \\
\text { Bagkhor }\end{array}$ & 2458 & Herb & Pink & $\begin{array}{l}\text { August- } \\
\text { October }\end{array}$ & GG116 & $\mathrm{A}$ \\
\hline Globba clarkei Baker [Zingiberaceae] & $\begin{array}{l}\text { Near Dobato } \\
\text { Kharka }\end{array}$ & 2278 & Climber & Orange & $\begin{array}{l}\text { August- } \\
\text { September }\end{array}$ & GG92 & $\mathrm{A}$ \\
\hline $\begin{array}{l}\text { Holboellia latifolia Wall. } \\
\text { [Berberidaceae] }\end{array}$ & $\begin{array}{l}\text { Between naag } \\
\text { Pokhari } \\
\text { Kharka and } \\
\text { Bisauni }\end{array}$ & 3060 & Climber & $\begin{array}{l}\text { Pale } \\
\text { Green }\end{array}$ & $\begin{array}{l}\text { September- } \\
\text { October }\end{array}$ & GG44 & $\mathrm{R}$ \\
\hline $\begin{array}{l}\text { Hoya lanceolata Wall. ex D.Don } \\
\text { [Apocynaceae] }\end{array}$ & $\begin{array}{l}\text { Between } \\
\text { Milinge and } \\
\text { Kaldobensi }\end{array}$ & 1492 & Climber & White & April-May & GG58 & $\mathrm{R}$ \\
\hline $\begin{array}{l}\text { Hydrangea anomala D Don } \\
\text { [Hydrangeaceae] }\end{array}$ & $\begin{array}{l}\text { Near } \\
\text { Baagkhor } \\
\end{array}$ & 2475 & Vine & $\begin{array}{l}\text { Light } \\
\text { Green }\end{array}$ & April-May & GG54 & $\mathrm{R}$ \\
\hline $\begin{array}{l}\text { Hypericum choisianum Wall. ex } \\
\text { N.Robson [Hypericaceae] }\end{array}$ & $\begin{array}{l}\text { On the way to } \\
\text { Dobato } \\
\text { Kharka }\end{array}$ & 2278 & Shrub & Yellow & $\begin{array}{l}\text { August- } \\
\text { September }\end{array}$ & GG89 & $\mathrm{R}$ \\
\hline $\begin{array}{l}\text { Hypericum uralum Buch. -Ham. ex D. } \\
\text { Don [Hypericaceae] }\end{array}$ & $\begin{array}{l}\text { Dhaunlodand } \\
\text { a }\end{array}$ & 2067 & Shrub & Yellow & $\begin{array}{l}\text { August- } \\
\text { September }\end{array}$ & GG85 & A \\
\hline $\begin{array}{l}\text { Impatiens bicornuta Wall. } \\
\text { [Balsaminaceae] }\end{array}$ & $\begin{array}{l}\text { On the way to } \\
\text { Bagkhor }\end{array}$ & 2418 & Herb & Yellow & July-September & GG98 & A \\
\hline $\begin{array}{l}\text { Impatiens puberula } \mathrm{DC} . \\
\text { [Balsaminaceae] }\end{array}$ & $\begin{array}{l}\text { Ghyachowk } \\
\text { village }\end{array}$ & 1746 & Herb & Purple & July-September & GG12 & A \\
\hline $\begin{array}{l}\text { Indigofera exilis Grierson \& } \\
\text { D.G.Long [Leguminosae] }\end{array}$ & $\begin{array}{l}\text { Between } \\
\text { Poduchautara } \\
\text { and Baagkhor }\end{array}$ & 2537 & Shrub & Purple & April -June & GG53 & $\mathrm{A}$ \\
\hline $\begin{array}{l}\text { Juncus wallichianus J.Gay ex Laharpe } \\
\text { [Juncaceae] }\end{array}$ & $\begin{array}{l}\text { Way to } \\
\text { Nagepokhri }\end{array}$ & 3189 & Herb & White & $\begin{array}{l}\text { August- } \\
\text { October }\end{array}$ & GG117 & $\mathrm{R}$ \\
\hline $\begin{array}{l}\text { Leea macrophylla Roxb. ex Hornem. } \\
\text { [Vitaceae] }\end{array}$ & Saalghari & 1037 & Herb & White & $\begin{array}{l}\text { August - } \\
\text { September }\end{array}$ & GG66 & $\mathrm{A}$ \\
\hline $\begin{array}{l}\text { Leucas cephalotes (Roth) Spreng. } \\
\text { [Lamiaceae] }\end{array}$ & Saalghari & 1037 & Herb & White & $\begin{array}{l}\text { August- } \\
\text { September }\end{array}$ & GG65 & $\mathrm{R}$ \\
\hline $\begin{array}{l}\text { Marsdenia lucida } \text { Edgew. ex } \\
\text { Maddden [Apocynaceae] }\end{array}$ & $\begin{array}{l}\text { On the way to } \\
\text { Bagkhor }\end{array}$ & 2583 & Climber & White & $\begin{array}{l}\text { August- } \\
\text { September }\end{array}$ & GG134 & $\mathrm{R}$ \\
\hline $\begin{array}{l}\text { Mazus surculosus D. Don } \\
\text { [Phrymaceae] }\end{array}$ & $\begin{array}{l}\text { Ghyachowk } \\
\text { village }\end{array}$ & 1695 & Herb & Purple. & April-June & GG11 & A \\
\hline $\begin{array}{l}\text { Melastoma malabathricum } \mathrm{L} \text {. } \\
\text { [Melastomataceae] }\end{array}$ & $\begin{array}{l}\text { Dharapani, } \\
\text { Ghyachowk }\end{array}$ & 1700 & Shrub & Pink & April- June & GG6 & A \\
\hline $\begin{array}{l}\text { Mimosa rubicaulis Lam. } \\
\text { [Leguminosae] }\end{array}$ & Saalghari & 1037 & Tree & White & $\begin{array}{l}\text { August- } \\
\text { September }\end{array}$ & GG68 & A \\
\hline Nicotiana tabaccum L. [Solanaceae] & Ghyachchok & 1686 & Shrub & Pink & $\begin{array}{l}\text { August- } \\
\text { September }\end{array}$ & GG82 & A \\
\hline
\end{tabular}


Neera Joshi Pradhan \& Nirmala Joshi 49

\begin{tabular}{|c|c|c|c|c|c|c|c|}
\hline Scientific name & Locality & $\begin{array}{l}\text { Altitud } \\
\text { e (m) }\end{array}$ & Habit & $\begin{array}{l}\text { Flower } \\
\text { colour }\end{array}$ & Flowering time & $\begin{array}{l}\text { Collec- } \\
\text { tion } \\
\text { no. }\end{array}$ & Status \\
\hline Nicotiana tabaccum L. [Solanaceae] & Ghyachchok & 1686 & Shrub & Pink & $\begin{array}{l}\text { August- } \\
\text { September }\end{array}$ & GG82 & $\mathrm{A}$ \\
\hline $\begin{array}{l}\text { Oberonia pyrulifera Lindl. } \\
\text { [Orchidaceae] }\end{array}$ & $\begin{array}{l}\text { Near } \\
\text { Ghyachowk } \\
\text { village }\end{array}$ & & Herb & White. & April -July & Photo & App.II \\
\hline $\begin{array}{l}\text { Ophiopogon intermedius D.Don } \\
\text { [Asparagaceae] }\end{array}$ & $\begin{array}{l}\text { Near } \\
\text { Ghyachowk }\end{array}$ & 1700 & Herb & White & April- June & GG7 & $\mathrm{R}$ \\
\hline $\begin{array}{l}\text { Osbeckia nepalensis Hook.f. } \\
\text { [Melastomataceae] }\end{array}$ & Ghayachok & 1686 & Shrub & White & $\begin{array}{l}\text { August - } \\
\text { September } \\
\end{array}$ & GG136 & $\mathrm{A}$ \\
\hline $\begin{array}{l}\text { Oxygraphis polypetala (Raf.) Hook.f. } \\
\& \text { Thomson [Ranunculaceae] }\end{array}$ & $\begin{array}{l}\text { Near Nage } \\
\text { Pokhari }\end{array}$ & 3250 & Herb & Yellow & April-August & GG39 & $\mathrm{A}$ \\
\hline $\begin{array}{l}\text { Oxyspora paniculata (D. Don) DC. } \\
\text { [Melastomataceae] }\end{array}$ & $\begin{array}{l}\text { Milinge to } \\
\text { Ghyachchok }\end{array}$ & 1635 & Shrub & Pink & July-September & GG71 & $\mathrm{R}$ \\
\hline $\begin{array}{l}\text { Panisea uniflora (Lindl.) Lindl. } \\
\text { [Orchidaceae] }\end{array}$ & $\begin{array}{l}\text { Between } \\
\text { Milinge and } \\
\text { Kaldobensi }\end{array}$ & 1277 & Herb & $\begin{array}{l}\text { Light } \\
\text { Yellowi } \\
\text { sh }\end{array}$ & Aapril-June & $\begin{array}{l}\text { Sketch/ } \\
\text { Photo }\end{array}$ & App.II \\
\hline $\begin{array}{l}\text { Parnassia pusilla Wall. ex Arn. } \\
\text { [Celastraceae] }\end{array}$ & Deurali & 3360 & Herb & White & July-September & GG124 & $\mathrm{R}$ \\
\hline $\begin{array}{l}\text { Pavetta tomentosa Roxb. ex Sm. } \\
\text { [Rubiaceae] }\end{array}$ & $\begin{array}{l}\text { Near } \\
\text { Kaldobensi }\end{array}$ & 1267 & Shrub & White. & May-september & GG60 & $\mathrm{R}$ \\
\hline $\begin{array}{l}\text { Pedicularis megalantha D.Don } \\
\text { [Scrophulariaceae] }\end{array}$ & Deurali & 3303 & Herb & Pink & July -September & GG133 & $\mathrm{R} / \mathrm{HE}$ \\
\hline $\begin{array}{l}\text { Pleione hookeriana (Lindl.) Rollisson } \\
\text { [Orchidaceae] }\end{array}$ & & 3081 & Herb & $\begin{array}{l}\text { Light } \\
\text { Purple }\end{array}$ & April-June & $\begin{array}{l}\text { Illustra } \\
\text { tion }\end{array}$ & App.II \\
\hline $\begin{array}{l}\text { Polygala arillata Buch.-Ham. ex D. } \\
\text { Don [Polygalaceae] }\end{array}$ & $\begin{array}{l}\text { On the way to } \\
\text { Bagkhor }\end{array}$ & 2418 & Tree & Yellow & $\begin{array}{l}\text { July - } \\
\text { September } \\
\end{array}$ & GG100 & $\mathrm{R}$ \\
\hline $\begin{array}{l}\text { Polygonum molle D.Don } \\
\text { [Polygonaceae] }\end{array}$ & $\begin{array}{l}\text { Near Dobato } \\
\text { Kharka }\end{array}$ & 2278 & Herb & $\begin{array}{l}\text { Whitish } \\
\text { pink }\end{array}$ & $\begin{array}{l}\text { July- } \\
\text { Septempber }\end{array}$ & GG94 & $\mathrm{A}$ \\
\hline $\begin{array}{l}\text { Potentilla peduncularis D.Don } \\
\text { [Rosaceae] }\end{array}$ & $\begin{array}{l}\text { Dhaunlodand } \\
\text { a }\end{array}$ & 2067 & Herb & Yellow & June-October & GG87 & A \\
\hline Primula denticulata Sm. [Primulceae] & $\begin{array}{l}\text { Near Nage } \\
\text { Pokhari } \\
\end{array}$ & 3250 & Herb & Purple & March-May & GG38 & $\mathrm{R}$ \\
\hline $\begin{array}{l}\text { Prunus cornuta (Wall. ex Royle) } \\
\text { Steud. [Rosaceae] }\end{array}$ & $\begin{array}{l}\text { Near } \\
\text { Poduchautara }\end{array}$ & 2688 & Tree & White. & March-May & GG51 & $\mathrm{R}$ \\
\hline $\begin{array}{l}\text { Randia tetrasperma (Wall. ex Roxb.) } \\
\text { Benth. \& Hook.f. ex Brandis } \\
\text { [Rubiaceae] }\end{array}$ & $\begin{array}{l}\text { Between } \\
\text { Dhodke } \\
\text { pokhari and } \\
\text { Ghyachowk }\end{array}$ & 1802 & Shrub & $\begin{array}{l}\text { Creamy } \\
\text { White }\end{array}$ & March-May & GG23 & $\mathrm{R}$ \\
\hline $\begin{array}{l}\text { Ranunculus hirtellus Royle } \\
\text { [Ranunculaceae] }\end{array}$ & & 1910 & Herb & Yellow & April-June & GG15 & $\mathrm{A}$ \\
\hline $\begin{array}{l}\text { Remusatia hookeriana } \text { Schott } \\
\text { [Araceae] }\end{array}$ & $\begin{array}{l}\text { Near Dhodke } \\
\text { Pokhari }\end{array}$ & 2058 & Herb & Yellow & April-June & GG19 & $\mathrm{A}$ \\
\hline $\begin{array}{l}\text { Rhododendron arboreum } \mathrm{Sm} . \\
\text { [Ericaceae] }\end{array}$ & $\begin{array}{l}\text { Between } \\
\text { Baagkhor and } \\
\text { Nage Pokhari } \\
\text { Kharka }\end{array}$ & 2984 & Tree & White & April-June & GG32 & A \\
\hline $\begin{array}{l}\text { Rhododendron dalhousieae Hook.f. } \\
\text { [Ericaceae] }\end{array}$ & $\begin{array}{l}\text { Damara, } \\
\text { Ghyachok }\end{array}$ & 2323 & Shrub & White & April-June & Sketch & $\mathrm{R}$ \\
\hline $\begin{array}{l}\text { Rhynchostylis retusa (L.) Blume } \\
\text { [Orchidaceae] }\end{array}$ & $\begin{array}{l}\text { Salghari: } \\
\text { Between } \\
\text { Baluwa and } \\
\text { Kaldobensi }\end{array}$ & 1078 & Herb & Purple & May-October & Photo & App.II \\
\hline Rosa brunonii Lindl. [Rosaceae] & $\begin{array}{l}\text { Between } \\
\text { Dhodke } \\
\text { pokhari and } \\
\text { Ghyachowk }\end{array}$ & 1938 & Vine & White & April-June & GG22 & $\mathrm{A}$ \\
\hline $\begin{array}{l}\text { Roscoea purpurea } \text { Smith } \\
\text { [Zingiberaceae] }\end{array}$ & $\begin{array}{l}\text { On the way to } \\
\text { Bagkhor }\end{array}$ & 2458 & Herb & Purple & May-September & GG108 & $\mathrm{R}$ \\
\hline $\begin{array}{l}\text { Rubus hoffmeisterianus Kunth \& } \\
\text { Bouche [Rosaceae] }\end{array}$ & $\begin{array}{l}\text { Rambhanjyan } \\
\mathrm{g}\end{array}$ & 3261 & Creeper & $\begin{array}{l}\text { Pinkish } \\
\text { White }\end{array}$ & May-June & GG131 & $\mathrm{R}$ \\
\hline Rubus rugosus $\mathrm{Sm}$. [Rosaceae] & $\begin{array}{l}\text { Milinge to } \\
\text { Ghyachchok }\end{array}$ & 1635 & Climber & White & May-september & GG77 & $\mathrm{A}$ \\
\hline
\end{tabular}


50 Wild Flowers of Ghyachok VDC, Gorkha District, Nepal

\begin{tabular}{|c|c|c|c|c|c|c|c|}
\hline Scientific name & Locality & $\begin{array}{l}\text { Altitud } \\
\text { e (m) }\end{array}$ & Habit & $\begin{array}{l}\text { Flower } \\
\text { colour }\end{array}$ & Flowering time & $\begin{array}{l}\text { Collec- } \\
\text { tion } \\
\text { no. }\end{array}$ & Status \\
\hline Rubus treutleri Hook.f. [Rosaceae] & $\begin{array}{l}\text { Near Dobato } \\
\text { Kharka }\end{array}$ & 2278 & Climber & White & May-July & GG93 & $\mathrm{A}$ \\
\hline $\begin{array}{l}\text { Rumex nepalensis Spreng. } \\
\text { [Polygonaceae] }\end{array}$ & $\begin{array}{l}\text { Near } \\
\text { Ghyachowk }\end{array}$ & 1700 & Herb & $\begin{array}{l}\text { Dark } \\
\text { Red }\end{array}$ & May -June & GG8 & $\mathrm{A}$ \\
\hline $\begin{array}{l}\text { Sarcococca wallichii Stapf } \\
\text { [Buxaceae] }\end{array}$ & $\begin{array}{l}\text { On the way to } \\
\text { Dobato } \\
\text { Kharka }\end{array}$ & 2358 & Shrub & White & April-June & GG106 & $\mathrm{A}$ \\
\hline $\begin{array}{l}\text { Satyrium nepalense D.Don } \\
\text { [Orchidaceae] }\end{array}$ & $\begin{array}{l}\text { On the way to } \\
\text { Bagkhor }\end{array}$ & 2418 & Herb & Pink & August-October & Photo & App.II \\
\hline $\begin{array}{l}\text { Saxifraga strigosa Wall.ex Ser. } \\
\text { [Saxifragceae] }\end{array}$ & $\begin{array}{l}\text { Way to } \\
\text { Nagepokhri }\end{array}$ & 3189 & Herb & Yellow & $\begin{array}{l}\text { August- } \\
\text { September }\end{array}$ & GG112 & $\mathrm{R}$ \\
\hline $\begin{array}{l}\text { Saxifraga parnassifolia } \text { D. Don } \\
\text { [Saxifragceae] }\end{array}$ & $\begin{array}{l}\text { On the way to } \\
\text { Bagkhor }\end{array}$ & 2458 & Herb & Yellow & $\begin{array}{l}\text { September } \\
\text { october }\end{array}$ & GG114 & $\mathrm{R}$ \\
\hline $\begin{array}{l}\text { Schisandra grandiflora (Wall.) } \\
\text { Hook.f. \& Thomson [Schisandrceae] }\end{array}$ & $\begin{array}{l}\text { Between naag } \\
\text { Pokhari } \\
\text { Kharka and } \\
\text { Bisauni }\end{array}$ & 3081 & Vine & White & April-May & GG43 & $\mathrm{R}$ \\
\hline $\begin{array}{l}\text { Senecio graciliflorus DC. } \\
\text { [Compositae] }\end{array}$ & $\begin{array}{l}\text { Rambhanjyan } \\
\mathrm{g}\end{array}$ & 3264 & Herb & Yellow & August-October & GG121 & $\mathrm{R}$ \\
\hline $\begin{array}{l}\text { Setaria parviflora (Poir.) } \\
\text { M.Kerguelen [Poaceae] }\end{array}$ & Ghyachchok & 1686 & Herb & $\begin{array}{l}\text { Brownish } \\
\text { Green }\end{array}$ & Agust -October & GG83 & $\mathrm{A}$ \\
\hline $\begin{array}{l}\text { Sibbaldia sikkimensis (Prain) } \\
\text { Chatterjee [Rosaceae] }\end{array}$ & $\begin{array}{l}\text { Between } \\
\text { Baagkhor and } \\
\text { Nage Pokhari } \\
\text { Kharka }\end{array}$ & 2731 & Herb & Yellow. & April -June & GG30 & $\mathrm{R}$ \\
\hline $\begin{array}{l}\text { Smilax menispermoidea A.DC. } \\
\text { [Smilacaceae] }\end{array}$ & $\begin{array}{l}\text { Dobato } \\
\text { Kharka to } \\
\text { Bagkhor }\end{array}$ & 2278 & Climber & $\begin{array}{l}\text { White } \\
\text { Fruits } \\
\text { green }\end{array}$ & $\begin{array}{l}\text { August - } \\
\text { September }\end{array}$ & GG 92 & $\mathrm{~A}$ \\
\hline $\begin{array}{l}\text { Smitinandia micrantha (Lindl.) } \\
\text { Holttum [Orchidaceae] }\end{array}$ & $\begin{array}{l}\text { Salghari: } \\
\text { Between } \\
\text { Baluwa and } \\
\text { Kaldobensi }\end{array}$ & 1108 & Herb & Pink & March-May & Photo & App.II \\
\hline $\begin{array}{l}\text { Spiranthes sinensis (Pers.) Ames } \\
\text { [Orchidaceae] }\end{array}$ & $\begin{array}{l}\text { On the way to } \\
\text { Dobato } \\
\text { Kharka }\end{array}$ & 2278 & Herb & Pink & March-May & Photo & App.II \\
\hline $\begin{array}{l}\text { Swertia angustifolia } \text { Buch.-Ham. ex } \\
\text { D. Don [Gentianaceae] }\end{array}$ & $\begin{array}{l}\text { Milinge to } \\
\text { Ghyachchok }\end{array}$ & 1635 & Herb & $\begin{array}{l}\text { White } \\
\text { with } \\
\text { Purple } \\
\text { spot }\end{array}$ & September & GG78 & $\mathrm{E}$ \\
\hline $\begin{array}{l}\text { Symplocos ramosissima Wall. ex G. } \\
\text { Don [Symplocaceae] }\end{array}$ & $\begin{array}{l}\text { Between } \\
\text { Dhodke } \\
\text { pokhari and } \\
\text { Ghyachowk }\end{array}$ & 1938 & Tree & White & May -June & GG21 & $\mathrm{R}$ \\
\hline Tainia minor Hook.f. [Orchidaceae] & $\begin{array}{l}\text { Between } \\
\text { Dhodke } \\
\text { Pokhari and } \\
\text { Ghyachowk }\end{array}$ & 2023 & Herb & Yellow & April-May & Photo & App.II \\
\hline Taxus wallichiana Zucc. [Taxaceae] & $\begin{array}{l}\text { Between naag } \\
\text { Pokhari } \\
\text { Kharka and } \\
\text { Bisauni }\end{array}$ & 3081 & Tree & White & March-May & Photo & $\begin{array}{l}\text { App.II } \\
/ \mathrm{C} \mathrm{E}\end{array}$ \\
\hline $\begin{array}{l}\text { Thalictrum foliolosum DC. } \\
\text { [Ranunculaceae] }\end{array}$ & $\begin{array}{l}\text { Milinge to } \\
\text { Ghyachchok }\end{array}$ & 1635 & Herb & $\begin{array}{l}\text { Yellowish } \\
\text { Green }\end{array}$ & $\begin{array}{l}\text { August- } \\
\text { September }\end{array}$ & GG73 & A \\
\hline $\begin{array}{l}\text { Thalictrum reniforme Wall. } \\
\text { [Ranunculaceae] }\end{array}$ & $\begin{array}{l}\text { On the way to } \\
\text { Bagkhor }\end{array}$ & 2458 & Herb & Pink & $\begin{array}{l}\text { August- } \\
\text { September }\end{array}$ & GG115 & A \\
\hline $\begin{array}{l}\text { Tripterospermum volubile (D. Don) H. } \\
\text { Hara [Gentianaceae] }\end{array}$ & $\begin{array}{l}\text { On the way to } \\
\text { Bagkhor }\end{array}$ & 2458 & Climber & $\begin{array}{l}\text { Purplish } \\
\text { White }\end{array}$ & $\begin{array}{l}\text { August- } \\
\text { September }\end{array}$ & GG120 & A \\
\hline Triumfetta pilosa Roth [Malvaceae] & Saalghari & 1037 & Herb & Yellow & April-May & GG63 & $\mathrm{A}$ \\
\hline Urena lobata L. [Malvaceae] & Saalghari & 1037 & Shrub & Pink & April -May & GG64 & A \\
\hline $\begin{array}{l}\text { Viburnum erubescens Wall. } \\
\text { [Adoxaceae] }\end{array}$ & $\begin{array}{l}\text { Damara, } \\
\text { Ghyachok }\end{array}$ & 2371 & Shrub & White & April-May & GG31 & A \\
\hline Viola canescens Wall. [Violaceae] & $\begin{array}{l}\text { Baagkhor - } \\
\text { Nage Pokhari } \\
\text { Kharka }\end{array}$ & 2731 & Herb & Purple & April-May & GG40 & $\mathrm{R}$ \\
\hline Vitex negundo L [Lamiaceae] & $\begin{array}{l}\text { Above } \\
\text { Baluwa Bazar }\end{array}$ & 921 & Shrub & $\begin{array}{l}\text { Light } \\
\text { Purple. }\end{array}$ & April-May & GG61 & A \\
\hline
\end{tabular}


The most interesting of this field study was also to record the attractive flowers of the epiphytic Rhododendron dalhousiae on the Quercus species tree of which, the line drawing of this epiphyte species of Rhododendron dalhousiae is given in Figure 5. Additionally 8 species of epiphytes of Orchids are also recorded.

Among 119 wild flowers species, 4 species such as Taxus wallichiana, Allium wallichii, Swertia angustifolia, Arisaema costatum are placed critically endangered, threatened, endangered and least concern respectively (CAMP 2001; Ghimire et al. 2008). Similarly, out of 119 species, Orchid species and Taxus wallichiana and 11 species of Orchids are included in CITES Appendix II (Joshi et al. 2017). The line drawing of CITES species Pleione hookeriana is shown in Figure 6. The proper in situ and ex situ conservation and cultivation practice of these species may be helpful to conserve vulnerable, endangered, endemic and protected species.
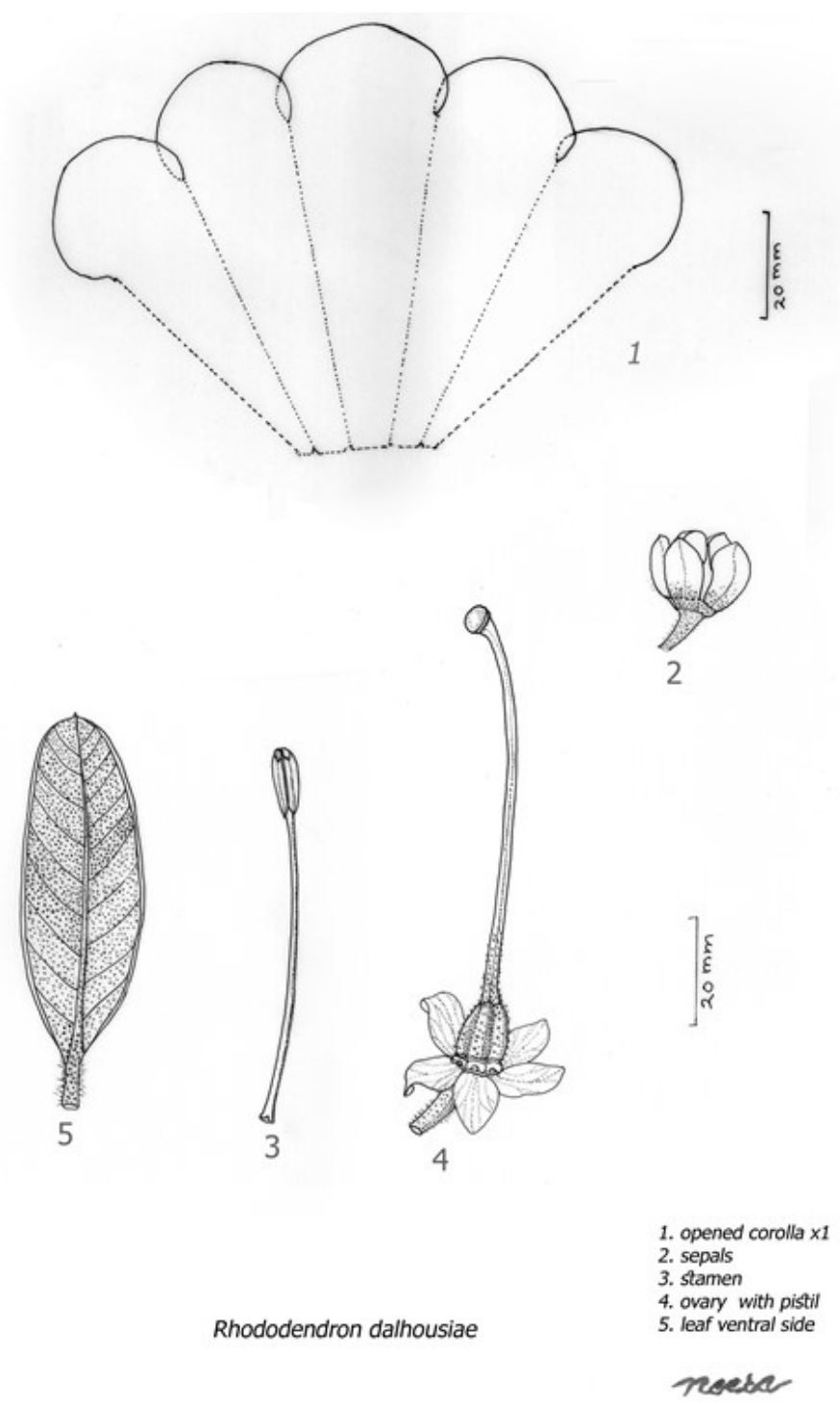

Figure 5. Line drawing of Rhododendron dalhousieae Hook.f. 


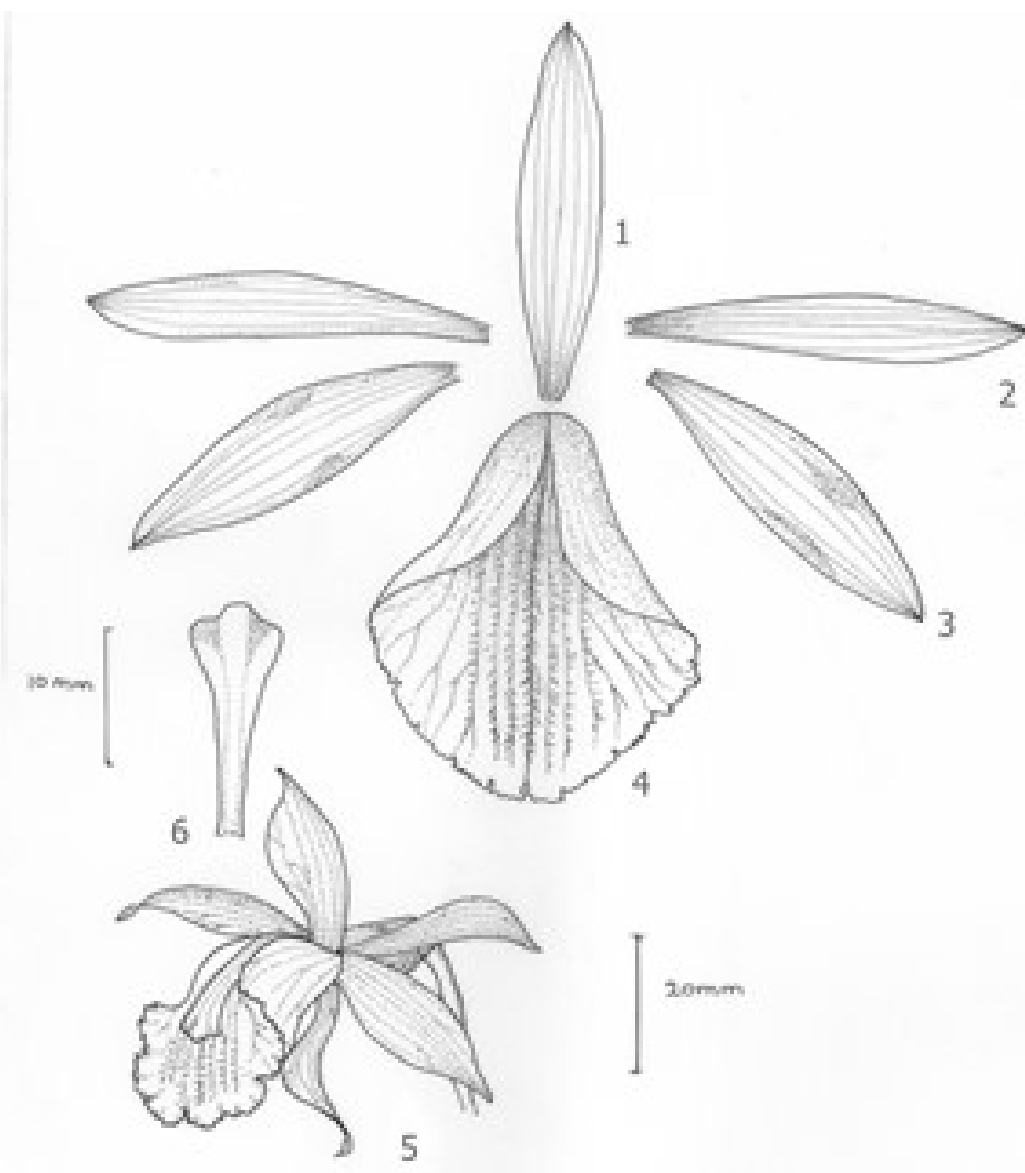

Pleione hookeriana

1.Dorsal sepal X 2, 2.Petal X 2, 3. Lateral sepal X 2, 4.Lip X 2 4.1p 5. side view of single flower X 1, 6.Column X2

Figure 6. Line drawing of CITES Appendix II species Pleione hookeriana (Lindl.) Rollisson

The most dominant flower colour is white with 41 species species. This is followed by yellow with 21 species, pink 12 species, purple 15 species, blue 4 species and light yellow, pinkish white with 3 species each, greenish yellow and light purple with 2 species each. There are many attractive beautiful flowers which may be utilized as ornamental, medicinal and edible value. So the the germplasm of this important species are to be conserved.

\section{Conclusion}

The checklist of the 119 flowering plants from Ghyachok VDC in two seasons is the debut collection from Ajirkot municipality, Gorkha district, belongs to 54 families of flowering plants. The result shows the collection is dominated by Orchidaceae which are placed in Appendix 
II CITES and other interesting families such as Ranunculceae, Rosaceae, Compositae, Leguminosae, Ericaceae, Polygonaceae, Berberidaceae, Begoniaceae, Gesneriaceae. The present information express the richness of flora of the study area ask for further researches in social, ethnobotanical, medicinal and commercial values. The urgent need to protect the CITES listed plants and other endangered plants are suggested to take conservation measures in the area by the concerned authorities. Hence the visual communication of such important plants would be one of the tools to bring awareness in identifying and protecting the rich heritage of the flora will definitely make a difference to local people, tourists, general people and policy makers. Hopefully concerned policy makers, scientists, botanists and explorer would add and take ahead further for study and research of the site.

\section{Acknowledgements}

Firstly our thanks goes to senior officers at Department of plants resources, Thapathali for sincere permission to collect and study the plant specimens of the Ghyachok VDC. Mr. Subash Khatri, Chief of NHPL, Scientific officer Ms. Rita Chettri, National Herbarium of Plants Laboratory (NHPL), Godavari for their kind cooperation. The study and trip to Ghyachok would not have been possible without the help from amazing local residents namely Ms. Nanda Kumari Gurung for generous home stays, Ms. Sapu Gurung for accompanying the whole field trip and Mr. Til Bahadur Gurung, Dhwoj Gurung for their kind guidance of the Ghychok village. We are thankful to Dr. B.N. Dhakal for designing the study sites map, and to our dedicated field team members Ms.Shanta Budha Magar, Ms.Sunita Shrestha and Ms. Sushila Sharma.

\section{LITERATURE CITED}

CAMP (Conservation Assessment \& Management Plan). 2001. Assessment \& Management Plan Workshop Report: Selected Medicinal Plant Species of Nepal, ed. V. Tandon,N. K. Bhattarai. MAPPA/IDRC;MOFSC; Nepal.

DHM (Department of Hydrology and Metrology) 2017. Climate data of Gorkha district. Department of Hydrology and Meteorology, Ministry of Energy, Water Resources and Irrigation, Kathmandu, Nepal.

DPG (District Profile of Gorkha), 2017. Published by Central bureau of statistics, Government of Nepal. National Planning Commission, CBS Gorkha division.

Ghimire, S.K., Sapkota, I..B., Oli, B.R., Parajuli, \& Rai, R. 2008. Non-Timber Forest Products of Nepal Himalaya: Database of Some Important Species Found in the Mountain Protected Areas and Surrounding Regions. $1^{\text {st }}$ edition. Kathmandu, Nepal: WWF.

Hara, H., Stearn, W.T. , \& Williams, L. H. J. 1978. An Enumeration of the Flowering Plants of Nepal, Vol. 1.British Museum of Natural History, London.

Hara, H. \& Williams, L.H.J.(Eds.).1979. An enumeration of the flowering plants of Nepal, Vol. 2. British Museum of Natural History, London.

Hara, H., Chater, A.O., \&Williams, L.H.J. (Eds.). 1982. An enumeration of the flowering plants of Nepal, Vol. 3. British Museum of Natural History, London.

Jain, S. K. \& Rao, R. R. 1977. A handbook of field and herbarium methods. Today \& Tomorrow's Printers and Publishers, New Delhi, India.

Joshi , N. 2011: Native Shrubs Suitable for Hedge Landscaping in Nepal. Bulletin of Department of Plant Resources, Thapathali, Kathmandu, Nepal. No. 33: pp.75-81. 
Joshi, N., Dhakal, K.S. \& Saud, D.S. 2017. Checklist of CITES Listed Flora of Nepal. Department of Plant Resources, Thapathali, Kathmandu, Nepal.

NBS (Nepal Biodiversity Strategy) 2002. Nepal Biodiversity Strategy, Ministry of Forests and Soil Conservation, supported by the Global Environmental Facility and UNDP, Kathmandu, Nepal.

Pearce, N.R. \& Cribb P.J. 2002. The Orchids of Bhutan. Vol 3 Part 3. Royal Botanic Garden, Edinburg andthe Royal Government of Bhutan.

Press, J.R., Shrestha, K.K.\& Sutton, D.A. 2000. Annotated checklist of the flowering plants of Nepal. The Natural History Museum, London.

Rajbhandari, K.R. \& Bhattarai, S. 2001.Beautiful Orchids of Nepal,Kathmandu, Nepal.

Rajbhandari, K.R. \& Adhikari, M.K. 2009. Endemic Flowering Plants of Nepal. Government of Nepal, Ministry of Forests and Soil Conservation, Department of Plant Resources, Thapathali, Kathmandu, Nepal, Part 3.

Rajbhandari, K.R., Bhattarai, K.R., \& Baral, S.R. (eds.) 2011. Catalogue of Nepalese Flowering Plants -II: Dicotyledons (Ranunculaceae to Dipsacaceae). Government of Nepal, National Herbarium and Plant Laboratoires, Department of Plant Resources, Godawari, Lalitpur, Nepal.

Rajbhandari, K.R., Bhattarai, K.R., \& Baral, S.R. (eds.) 2012. Catalogue of Nepalese Flowering Plants -III: Dicotyledons (Compositae to Salicaceae). Government of Nepal, National Herbarium and Plant Laboratoires, Department of Plant Resources, Godawari, Lalitpur, Nepal.

Rajbhandari, K.R. 2015. A Handbook of Orchids of Nepal. Government of Nepal. Ministry of Forest and Soil Conservation, Department of Plant Resources, Kathmandu, Nepal.

Rajbhandari, K.R., Rai, S.K., \& Bhatt, G.D. 2016. Endemic flowering plants of Nepal: An update. Bulletin Department of Plant Resources, Nepal. 38:106-144.

Raskoti, B.B. 2009. The Orchids of Nepal. Authors, Kathmandu.

Shrestha, T.B.\& Joshi, R.M. 1996. Rare, Endemic and Endangered Plants of Nepal. WWF Nepal Program, Kathmandu, Nepal.

Shrestha, K.K. Bhattarai, S.Bhandari, P. 2018. Handbook of Flowering Plants of Nepal.Vol 1. Gymnosperms and Angiosperms:Cycdaceae-Betulaceae.

Stainton, A. 1988. Flowers of the Himalaya- a supplemen.t. Oxford University Press, New Delhi, India.

Stainton, J.D.A. \& Polunin, O. 1984. Flowers of the Himalaya. Oxford University Press, New Delhi, India.

UNEP-WCMC 2004. Species data (unpublished) World Conservation Monitoring Centre of the United Nations Environment Programme (UNEP-WCMC). Cambridge, England Available online at: http://www.unep-wcmc.org

Watson, M. F, Akiyam, S., Ikeda, H. , Pendry, C., Rajbhandari, K. R. \& Shrestha, K.K. (eds.) 2011. Flora of Nepal.Vol 3. (Magnoliaceae-Rosaceae), Royal Botanic Garden, Edinburgh.

www.theplantlist.org 